\title{
Direct correlation of crystal structure and optical properties in wurtzite/zinc-blende GaAs nanowire heterostructures
}

\author{
Martin Heiss, ${ }^{1,2}$ Sonia Conesa-Boj, ${ }^{2,3}$ Jun Ren, ${ }^{4}$ Hsiang-Han Tseng, ${ }^{4}$ Adam Gali, ${ }^{5}$ Andreas Rudolph, ${ }^{6}$ \\ Emanuele Uccelli, ${ }^{1,2}$ Francesca Peiró, ${ }^{3}$ Joan Ramon Morante, ${ }^{7,3}$ Dieter Schuh, ${ }^{6}$ Elisabeth Reiger, ${ }^{6}$ \\ Efthimios Kaxiras, ${ }^{4}$ Jordi Arbiol, ${ }^{8}$ and Anna Fontcuberta i Morral ${ }^{1,2, *}$ \\ ${ }^{1}$ Walter Schottky Institut and Physik Department, Technische Universität München, Am Coulombwall 3, D-85748 Garching, Germany \\ ${ }^{2}$ Laboratoire des Matériaux Semiconducteurs, Institut des Matériaux, Ecole Polytechnique Fédérale de Lausanne, Lausanne, Switzerland \\ ${ }^{3}$ Departament d'Electrònica,Universitat de Barcelona, E-08028 Barcelona, Catalonia, Spain \\ ${ }^{4}$ Laboratory for Multiscale Modeling of Materials, Institut des Matériaux, Ecole Polytechnique Fédérale de Lausanne, Lausanne, Switzerland \\ ${ }^{5}$ Department of Atomic Physics, Budapest University of Technology and Economics, Budafoki út 8., H-1111, Budapest, Hungary \\ ${ }^{6}$ Institute for Experimental and Applied Physics, University of Regensburg, Universitätsstrasse 31, D-93053 Regensburg, Germany \\ ${ }^{7}$ Catalonia Institute for Energy Research (IREC), E-08019 Barcelona, Catalonia, Spain \\ ${ }^{8}$ Institució Catalana de Recerca i Estudis Avançats (ICREA) and Institut de Ciència de Materials de Barcelona, Consejo Superior de \\ Investigaciones Científicas (CSIC), E-08193 Bellaterra, Catalonia, Spain
}

(Received 12 July 2010; revised manuscript received 28 September 2010; published 20 January 2011)

\begin{abstract}
A method for the direct correlation at the nanoscale of structural and optical properties of single GaAs nanowires is reported. Nanowires consisting of $100 \%$ wurtzite and nanowires presenting zinc-blende/wurtzite polytypism are investigated by photoluminescence spectroscopy and transmission electron microscopy. The photoluminescence of wurtzite GaAs is consistent with a band gap of $1.5 \mathrm{eV}$. In the polytypic nanowires, it is shown that the regions that are predominantly composed of either zinc-blende or wurtzite phase show photoluminescence emission close to the bulk GaAs band gap, while regions composed of a nonperiodic superlattice of wurtzite and zinc-blende phases exhibit a redshift of the photoluminescence spectra as low as $1.455 \mathrm{eV}$. The dimensions of the quantum heterostructures are correlated with the light emission, allowing us to determine the band alignment between these two crystalline phases. Our first-principles electronic structure calculations within density functional theory, employing a hybrid-exchange functional, predict band offsets and effective masses in good agreement with experimental results.
\end{abstract}

DOI: 10.1103/PhysRevB.83.045303

PACS number(s): 78.55.Cr, 68.37.Og, 61.72.Mm, 78.67.Uh

\section{INTRODUCTION}

Semiconductor nanowires are attracting increasing interest because of their exciting optical and electronic properties and the possibility of synthesizing them in a controllable fashion. ${ }^{1-4}$ They are considered promising building blocks for the next generation of optical, sensing, electronic, and energy harvesting devices. ${ }^{5-8}$ Their small diameters, often at distances a few times the interatomic spacing in crystals, and their unique geometries lead to physical properties that differ significantly from the corresponding bulk material. Furthermore, nanowires hold the promise of integrating lattice-mismatched crystals on a single device, which opens new design possibilities and has the potential to significantly decrease production costs in solar cell applications. ${ }^{9}$

The functionality of nanowires is increased, and their properties are modified, when radial or axial heterostructures are created within a single wire. ${ }^{10,11}$ Radial heterostructures can be produced by growing different materials on the side facets of the nanowires, ${ }^{12,13}$ while axial heterostructures correspond generally to a variation in the chemical composition along the growth axis. ${ }^{2,4,14}$ Recently, a second type of axial heterostructure has attracted attention, where the crystalline structure varies along the growth axis while the chemical composition is conserved. Typically, the structure is changed from the cubic zinc-blende structure to the hexagonal wurtzite structure. ${ }^{15-17}$ This change of crystalline structure has been observed in some bulk materials such as $\mathrm{SiC}$ and $\mathrm{GaN}{ }^{18,19}$
In phosphides and arsenides, it has been shown that the appearance of the wurtzite phase is restricted to the nanowire form and is never observed in the bulk. ${ }^{20}$ The change in crystal structure is accompanied by changes in the electronic structure; for example, earlier theoretical work predicted a type-II band alignment between the zinc-blende and wurtzite phases in III-V semiconductors. ${ }^{21}$

Recently, type-II band alignment in InP nanowires ${ }^{22,23}$ has been demonstrated allowing the formation of crystal-phase quantum dots. ${ }^{24}$ Meanwhile, the band structure of wurtzite GaAs has been a subject of controversy in the past few years. Recent works on the luminescence of GaAs nanowires with a high percentage of wurtzite phase show a large variety of results. Recently, luminescence of wurtzite GaAs in the range of $1.53-1.54 \mathrm{eV}$ has been reported. ${ }^{25}$ Previously, Martelli et al. had reported an emission at $1.522 \mathrm{eV}$ in wurtzite GaAs nanostructures. ${ }^{26}$ Meanwhile Moewe et al. reported an emission at $10 \mathrm{meV}$ below the zinc-blende GaAs band gap. ${ }^{27}$ Additionally, our own results presenting a mixture of wurtzite and zinc-blende phases in nanowires are consistent with a type-II band alignment and smaller band gap. ${ }^{28}$ Indeed, in nanowires presenting $30 \%$ and $70 \%$ wurtzite phase we have observed extremely sharp peaks at 1.43 and $1.515 \mathrm{eV}^{28}$ The spectra could be explained by the existence of quantum heterostructures forming a type-II band alignment. ${ }^{28}$ Additionally, similar results have been obtained by other groups. ${ }^{24}$ We believe that the apparent contradictions of some of these literature results can be related to the fact that the 
(a)

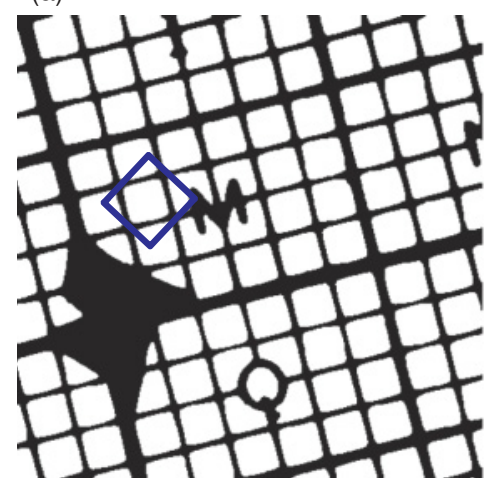

(b)

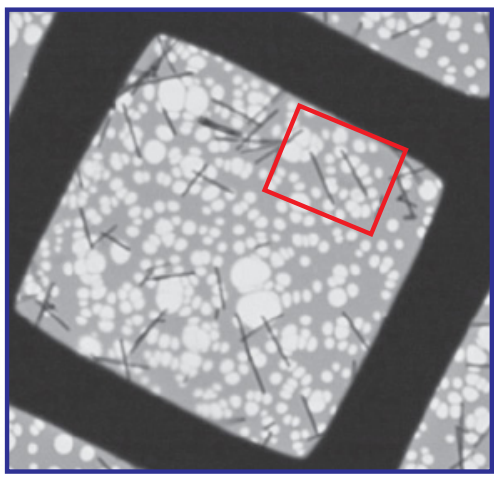

(c)

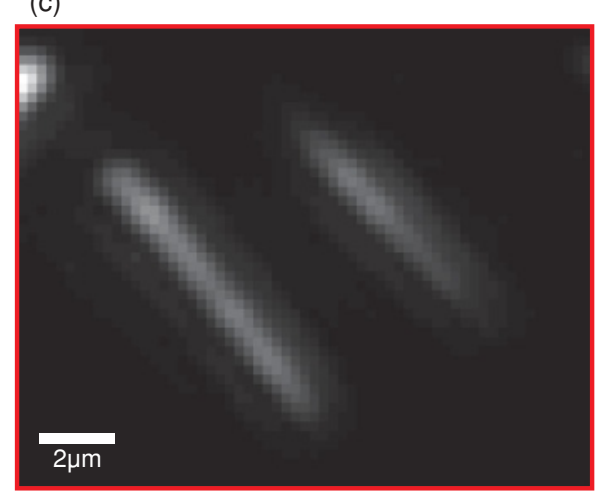

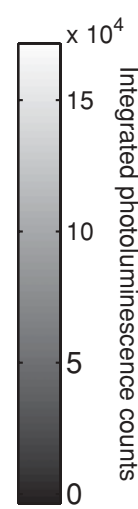

FIG. 1. (Color online) Overview of the measurement process: (a) Optical microscopy image of a TEM copper grid with marker symbols used for the studies. (b) Detail of TEM grid showing the nanowires transferred to the holey carbon film. (c) Intensity plot of integrated PL signal at $4.2 \mathrm{~K}$ from the area of the TEM grid marked by the box in (b).

optical and structural characterizations were not performed on the same nanowire. This limits the possibilities to account for variability in the structure of different nanowires from one growth. In this work we will perform a direct correlation between the structural and optical properties in order to overcome this limitation.

An initial effort in this direction was carried out by Bao et al., ${ }^{22}$ who looked at the shift of the luminescence band as a function of the excitation power for highly twinned and twin-free zinc-blende structures in InP nanowires. Arbiol et al. $^{29}$ demonstrated, by in situ local electron-energy-loss spectroscopy (EELS) measurements on [111] zinc-blende inclusions in [0001] wurtzite nanowires in $\mathrm{GaN}$, the variation in the local density of states close to the band gap. Finally, in different types of heterostructures, transmission electron microscopy (TEM) based cathodoluminescence was used to characterize GaN/InGaN/AlGaN nanowire heterostructures. ${ }^{30}$ This technique enabled the direct correlation between the structural quality and the carrier recombination characteristics in InGaN quantum wells. ${ }^{30}$

In this work, we provide a study that demonstrates a direct correlation between the optical properties and structure at the atomic scale of GaAs nanowires formed by either pure wurtzite GaAs or a combination of various thicknesses of the zinc-blende and wurtzite phases. This enables us to elucidate the band alignment between the two crystalline phases and represents an important step toward structural band-gap engineering.

\section{EXPERIMENTAL DETAILS}

The nanowires were grown in a Gen II molecular beam epitaxy (MBE) system. Wurtzite GaAs nanowires were grown by using gold as a catalyst, while the polytypic GaAs nanowires were obtained by the gold-free gallium-assisted growth method. In order to avoid cross contamination because of the use of gold, the two different samples were fabricated in separate MBE systems. The nanowires with $\sim 98 \%-100 \%$ wurtzite GaAs were grown on GaAs (1111)B substrates at a growth temperature of $540{ }^{\circ} \mathrm{C}$ under a $\mathrm{As}_{4}$ beam flux of $1.2 \times 10^{-6}$ mbar at a Ga rate equivalent to a planar growth of $0.8 \AA / \mathrm{s}$. The growth time was $7500 \mathrm{~s}$. The nucleation and growth followed the vapor-liquid-solid mechanism, with $\mathrm{Au}$ as the catalyst. ${ }^{31}$ Details on the growth procedure are described in Ref. 32. After the axial growth of the nanowires, the growth parameters were changed to conditions suitable for planar growth and the nanowires were passivated by an epitaxial prismatic shell of $\mathrm{AlGaAs} / \mathrm{GaAs}$ material. ${ }^{12,13}$ The two-dimensional (2D) equivalent amount grown during capping was $60 \mathrm{~nm}$ AlGaAs followed by $30 \mathrm{~nm} \mathrm{GaAs}$.

Wurtzite/zinc-blende polytypic nanowires were obtained on (111)B GaAs substrates previously coated with $20 \mathrm{~nm} \mathrm{SiO}$. $_{2}$ The growth temperature was $630^{\circ} \mathrm{C}$ and the As beam flux at the beginning of the growth process was $8.8 \times 10^{-7}$ mbar at a Ga rate equivalent to a planar growth of $0.22 \AA /$ s. For this sample, the As beam flux increased slightly up to $\sim 1 \times 10^{-6}$ mbar during the growth. The nanowires were capped by an epitaxial shell of AlGaAs/GaAs. ${ }^{12,13}$

For the direct correlation experiments, the nanowires were first transferred onto TEM holey carbon $\mathrm{Cu}$ grids. The grids presented markers in order to facilitate the localization of the nanowires in the multiple measurements [see Figs. 1(a) and 1(b)]. For the optical characterization, the grids were fixed freely suspended to a sample holder mounted in a confocal microphotoluminescence ( $\mu \mathrm{PL})$ setup. The sample stick was then immersed in a liquid-helium bath cryostat. In order to ensure a good thermal dissipation during the optical measurements, the sample space was filled with a helium exchange gas at a pressure of $\sim 5$ mbar. The $\mu \mathrm{PL}$ measurements were carried out at a temperature of $4.2 \mathrm{~K}$. The excitation sources were a semiconductor laser diode emitting at $780 \pm 10 \mathrm{~nm}$ or a helium neon laser emitting at $632.8 \mathrm{~nm}$. The laser was focused to a diffraction-limited spot of $\sim 0.8 \mu \mathrm{m}$ in diameter. This diffraction limit of light of the confocal microscopy is also the limiting factor for the overall resolution of the correlation technique. Typical excitation power densities were in the order of $10-50 \mathrm{~W} / \mathrm{cm}^{2}$, orders of magnitude below the level where significant heating of the sample could be observed (Fig. 2). Indeed, in contrast to similar experiments performed in a helium flow cryostat, ${ }^{22}$ we did not observe any severe heating effects resulting from the low heat conductivity of the thin carbon membrane. In our case, we are able to realize $\mu \mathrm{PL}$ measurements on nanowires dispersed to a freestanding carbon membrane without the 


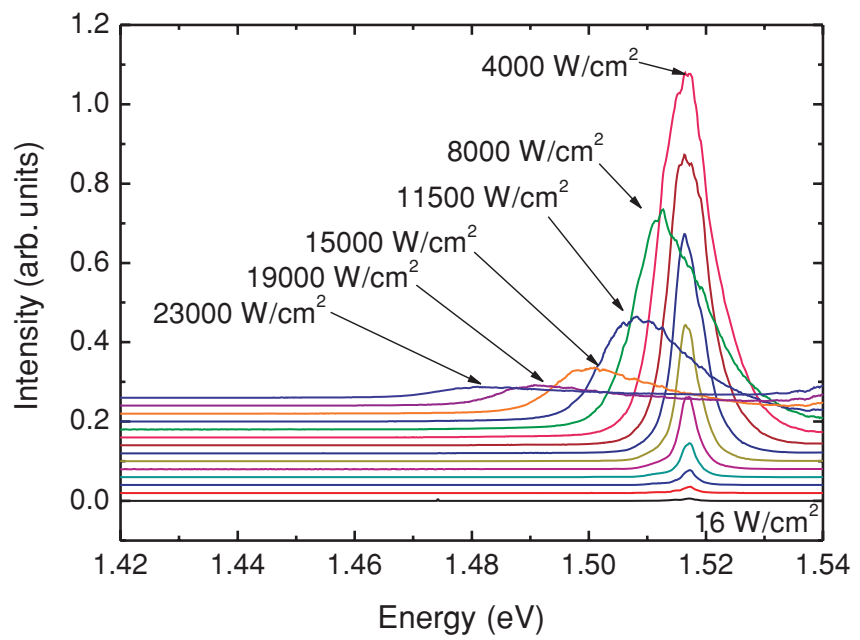

FIG. 2. (Color online) Excitation power dependence of a typical capped zinc-blende GaAs nanowire, dispersed on a holey carbon film of a copper TEM grid. No pronounced heating effects are observed for excitation power densities up to $4000 \mathrm{~W} / / \mathrm{m}^{2}$. For higher excitation powers the heating of the sample causes a change in band gap, resulting in a redshift of the PL transition. For the highest excitation of $23000 \mathrm{~W} / \mathrm{cm}^{2}$, the redshift corresponds to a temperature increase from 4.2 to $140 \mathrm{~K}$. This clearly demonstrates that heating can be neglected for measurements performed at excitation powers in the order of $10-50 \mathrm{~W} / \mathrm{cm}^{2}$.

need of adding supplementary layers on top to allow for thermal dissipation as it was realized in previous studies. ${ }^{22}$ Only this configuration enables simultaneous high-resolution TEM (HRTEM) and $\mu$ PL measurements on the same single nanowire. The position of the sample in the focal plane of the objective was verified by acquiring a confocal reflectivity image from the sample area. The $\mu \mathrm{PL}$ measurements were then acquired by scanning an area of $20 \mu \mathrm{m} /$ side with a step of $250 \mathrm{~nm}$ and acquiring a spectrum at each position [see Fig. 1(c)]. The spatial dependence of PL spectra along the nanowire axis was obtained by plotting the suitable cross section of this dataset.

Immediately after $\mu \mathrm{PL}$ measurements the nanowires were studied by TEM. A series of connected bright-field HRTEM micrographs was obtained from the bottom to the top of the nanowire, with the purpose of obtaining detailed information on the structure. Both high- and low-resolution measurements were taken. The micrographs were taken along the nanowire, ensuring that the ends were overlapping with the previous and/or following micrographs. Multiple subsequent micrographs were necessary to map the whole length. The electron microscopy (TEM and HRTEM) measurements were carried out in a CM300 LaB6 microscope with a point-to-point resolution of $0.17 \mathrm{~nm}$.

\section{RESULTS}

\section{A. Wurtzite GaAs band gap}

We start by presenting results in nanowires consisting of nearly $100 \%$ wurtzite. In this case, the direct correlation method is less important, as there is no significant variation on the structure along the nanowire. The correlation allows us
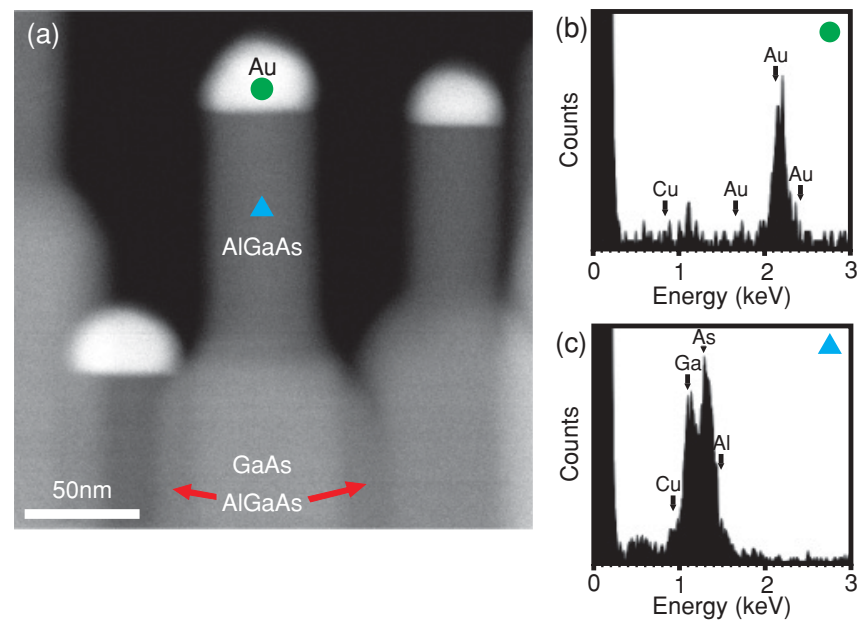

FIG. 3. (Color online) (a) Typical HAADF STEM micrograph obtained on a Au-seeded AlGaAs/GaAs nanowire. (b) EDX spectrum taken at the tip of the nanowire confirming the presence of $\mathrm{Au}$. (c) EDX spectrum at the neck of the nanowire confirming the presence of $\mathrm{AlGaAs}$ on the neck (the percentage of $\mathrm{Al}$ is low).

to verify that the PL characteristics are correctly attributed to nanowires composed from wurtzite GaAs.

Figure 3(a) shows a high-angle annular dark-field (HAADF) scanning TEM (STEM) micrograph of several nanowires from the sample. In principle, the HAADF detector directly gives a contrast proportional to the atomic charge $Z$ of the nuclei in the sample. The contrast also depends on the sample thickness that is not uniform in the axial extension compared to the rest of the wire. The image contrast in Fig. 3(a) therefore cannot directly indicate the chemical composition. For this reason energy-dispersive x-ray spectroscopy (EDX) was performed at the thin extension and the catalyst droplet to clearly determine the chemical composition. Figure 3(b) shows the EDX spectrum taken with the electron beam focused at the tip of the nanowire. The spectral features clearly demonstrate the presence of the $\mathrm{Au}$ catalyst. Meanwhile, Fig. 3(c) shows that the thin extension of the nanowire toward the tip corresponds to axially grown AlGaAs. This is in clear agreement with the picture that, during the radial capping with the $\mathrm{AlGaAs} / \mathrm{GaAs}$ shell, the nanowire continues to grow axially from precipitation below the catalyst droplet. Indeed, for the case of the Au-catalyzed nanowire, the metallic catalyst is not affected by the growth conditions. We can therefore use the size of the nanowire neck to accurately estimate the size of the nanowire core.

Now we turn to the correlation between HRTEM and spatially dependent PL. In Fig. 4 we have included TEM micrographs representing various locations along the nanowire. As shown at the left-hand end, the nanowire consists of pure wurtzite GaAs free of stacking faults throughout a $1.2-\mu \mathrm{m}-$ long region A (Fig. 4). In the 150-nm-long region B we can observe seven stacking faults in the nanowire that is otherwise still composed of pure wurtzite phase. The following $1 \mu \mathrm{m}$ of the nanowire (C) are stacking-fault-free wurtzite GaAs material. The final $250 \mathrm{~nm}$ (D) of the nanowire exhibits a mixture of heavily twinned zinc-blende and wurtzite phases. This can be attributed to the stage in which the conditions (mainly As 

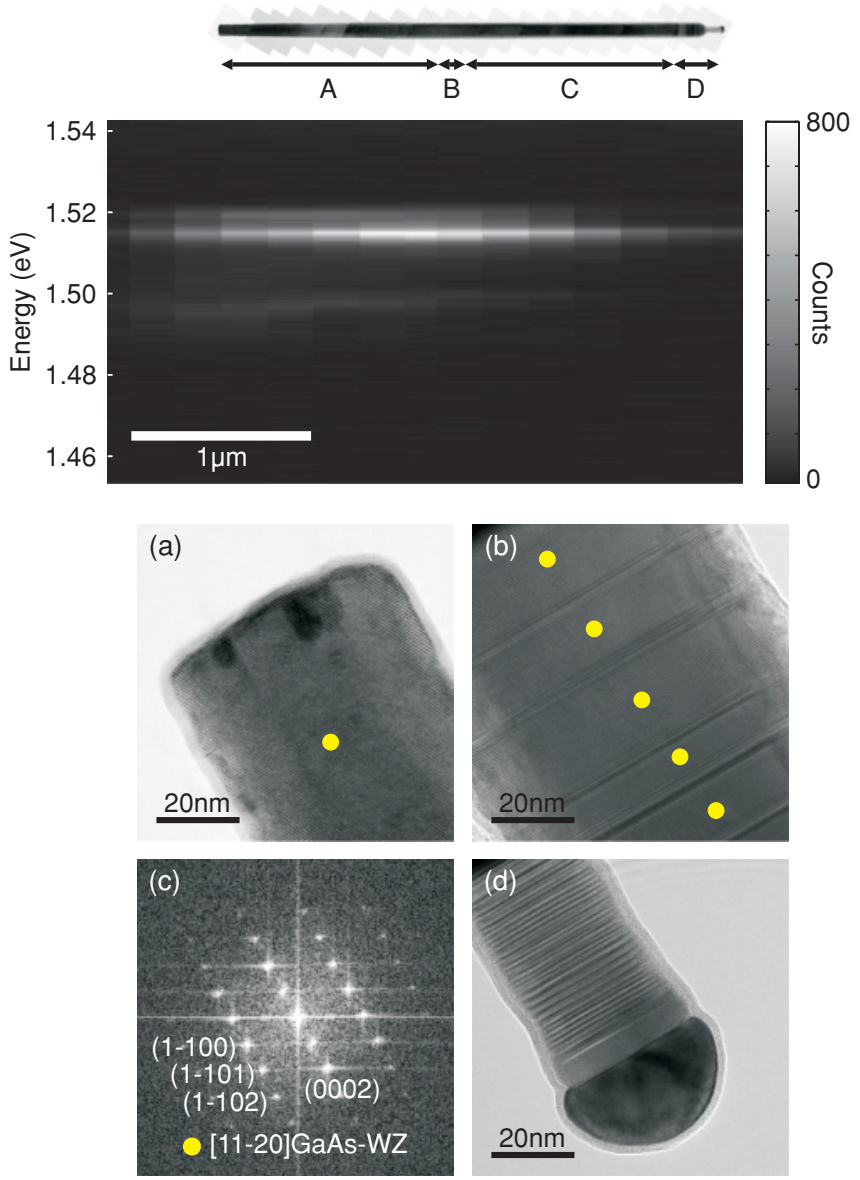

FIG. 4. (Color online) Mapping of PL spectra along the length of the nanowire shown above. The scale bar applies to both the PL mapping and the TEM. (a), (b), (d) HRTEM micrographs showing the crystalline phases at the regions of the nanowire indicated by the arrows above. Filled yellow circles mark the wurtzite GaAs phase. (c) Power spectrum of HRTEM micrograph in region c. Note that micrograph in region $\mathrm{D}$ corresponds to the axially grown $\mathrm{AlGaAs} / \mathrm{GaAs}$ material during the radial capping forming a thin extension.

pressure) were changed from axial to predominantly radial growth. Region D corresponds to the remaining axial growth occurring during the capping and is composed of AlGaAs, as discussed before. Figure 5 shows the typical PL spectra along the $\sim 2.8-\mu \mathrm{m}$-long nanowire. An emission peak at $\sim 1.515 \mathrm{eV}$ is observed throughout the length of the nanowire. By exciting the luminescence with a HeNe laser with a photon energy of $1.96 \mathrm{eV}$, we have verified that there is no emission in the range of energies between 1.52 and $1.63 \mathrm{eV}$. This is in agreement with our previous studies in which the luminescence in wurtzite/zinc-blende heterostructured nanowires was always observed below $1.515 \mathrm{eV}^{28}$ Furthermore, this value is in agreement with recent theoretical predictions. ${ }^{33}$

We have examined at least four nanowires with the same structural characteristics (nearly 100\% wurtzite) and we did not observe any luminescence at the energies between 1.52 and $1.63 \mathrm{eV}$. Additionally, in order to rule out possible contamination of the nanowires, we have measured the PL of a 1- $\mu \mathrm{m}$-thick GaAs epilayer. The spectrum is shown in Fig. 5.

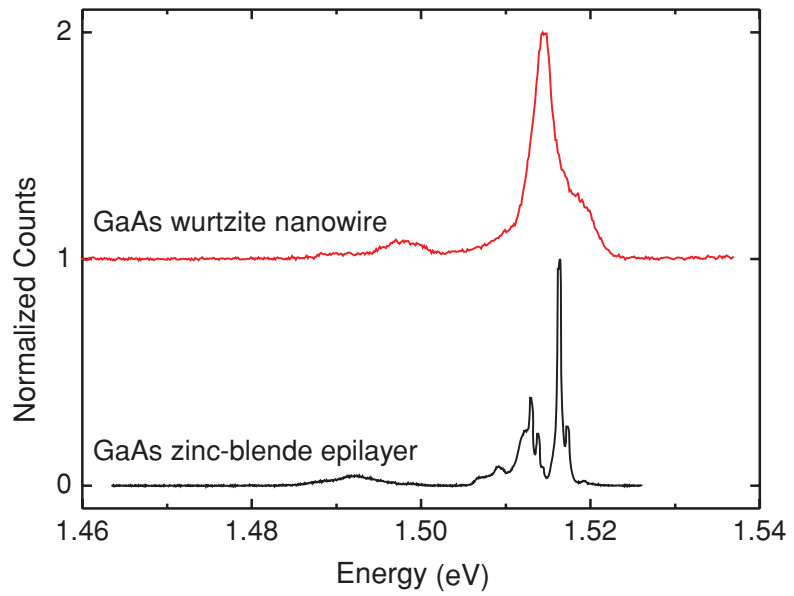

FIG. 5. (Color online) (Top) PL spectrum from a nanowire consisting of pure wurtzite GaAs phase. (Bottom) PL of a planar zinc-blende GaAs reference sample.

The emission is peaked at $1.516 \mathrm{eV}$, as expected for excitonic transitions in high-purity GaAs. The additional sharp peaks in the PL spectrum as well as the broad emission at $1.49 \mathrm{eV}$ can be attributed to carbon impurities. As a consequence, we can conclude that our measurements are consistent with an interband transition in the wurtzite GaAs at $1.515 \mathrm{eV}$, clearly originated in the core of the nanowire.

Finally, we want to further discuss the relation between the observed emission at $1.515 \mathrm{eV}$ and the band gap of bulk wurtzite GaAs. The diameter of the catalyst droplet and therefore the nanowire core is $\sim 33 \mathrm{~nm}$ [see Fig. 4(d)]. Owing to the small size, the radial confinement of carriers is causing a slight blueshift with respect to the actual bulk band gap. We have estimated this confinement energy by simulating ${ }^{34}$ this structure with nextnano, ${ }^{3}$ the next generation 3D nanodevice simulator. For simplicity, the calculations use the zinc-blende GaAs band parameters. The simulations show a confinement energy in the range of $13.5-17.8 \mathrm{meV}$ for diameters between 35 and $30 \mathrm{~nm}$. By correcting the observed emission energy with the estimated radial confinement energy, we obtain an estimated wurtzite GaAs band gap of $1.50 \mathrm{eV}$.

\section{B. Wurtzite/zinc-blende GaAs band offsets}

We now turn to the central aspect of this work, which is the correlation between the structural and optical properties in wurtzite/zinc-blende heterostructures. Representative SEM micrography of the as-grown Ga-catalyzed sample is shown in Fig. 6. The gallium droplet at the top of the nanowire disappeared during the growth of the shell.

Figure 7(a) shows the $\mu \mathrm{PL}$ spatial dependence in a 7- $\mu \mathrm{m}$ long nanowire with a diameter of $170 \mathrm{~nm}$. Below the PL scan we have included TEM micrographs representative of various locations on the nanowire. From the structural point of view, the nanowire is composed of two regions: (i) one with zincblende and a variation in twin density and (ii) one with a mixture of zinc-blende and wurtzite phases similar to a slightly disordered superlattice. The left-hand side of the nanowire is in the zinc-blende phase, with rotational twins separated by 


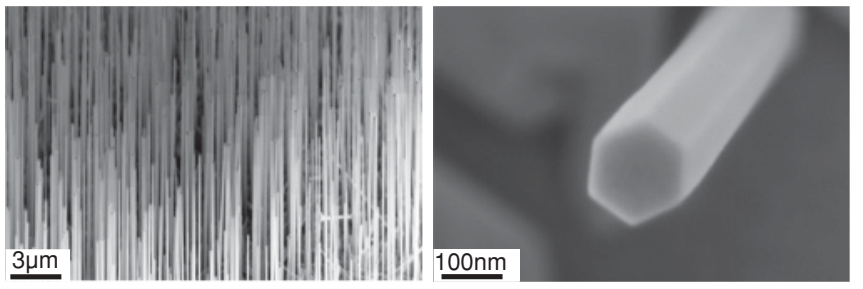

FIG. 6. SEMs of the as-grown nanowire sample grown with the Ga-assisted growth mechanism. The nanowires grow perpendicular to the $(1 \overline{1} 1) B$ substrate surface.

$\sim 100 \mathrm{~nm}$, shown in Fig. 7(b). From left to right, the density of deviations from an ideal zinc-blende crystal structure increases in a regular way.

Within $6 \mu \mathrm{m}$ from the left-hand end of the nanowire, these deviations are predominantly rotational twins and can be described considering a gradually increasing statistical twin density. For example, at positions 1,3 , and $5 \mu \mathrm{m}$, we observe a twin density of 60,223 and 479 twins/ $\mu \mathrm{m}$, respectively, shown in Figs. 7(c) and 7(d). This increase in the twin density is accompanied by a regular redshift of the PL peak from 1.50 to $1.48 \mathrm{eV}$. The PL signal is spatially discontinuous.

In the final third of the nanowire the concept of twin density is not sufficient to describe the structure of the nanowire, as segments of the wurtzite phase appear regularly. We can describe the structure by considering the fraction of the wurtzite phase present in the nanowire. At a distance of $6 \mu \mathrm{m}$ from the tip, the highly dense twin lattice turns into an alternation of 2-5 nm thick zinc-blende and wurtzite sections. At that point, the percentage of wurtzite is $\approx 50 \%$ and the average spacing of each of the phases is $\approx 2 \mathrm{~nm}$ [Fig. 7(e)]. Moving forward toward the right-hand side, the percentage of wurtzite quickly increases up to $100 \%, \sim 400 \mathrm{~nm}$ before the end of the nanowire. Some stacking faults and inclusions of zinc-blende sections smaller than $1 \mathrm{~nm}$ are observed right at the end, as shown in Fig. 7(f). A continuous wurtzite region of $290 \mathrm{~nm}$ is also found, shown in Fig. 7(g). This region is followed by a single 2.4-nm insertion of a zinc-blende phase followed by $84 \mathrm{~nm}$ of wurtzite. Exactly at the right end, we find a 46-nm-thick section with a mixture of wurtzite and zinc-blende phases.

Interestingly, for the nanowire section where the twin density increases, the PL shifts from 1.51 down to $1.48 \mathrm{eV}$. After this, consistent with the regions where wurtzite sections appear clearly, the PL peak shifts further down to $1.46 \mathrm{eV}$. At the final section of the nanowire a blueshift of the PL energy is observed. We never observe a PL signal above $1.515 \mathrm{eV}$. A total of four nanowires were investigated with the direct correlation method. They all showed a similar behavior. Another example is shown in Fig. 8(a). The measurements presented above raise two main questions:

(1) In the left-hand two-thirds of the nanowire, why is the twinning density shifting the PL emission toward lower energies from 1.51 to $1.48 \mathrm{eV}$ ?

(2) Can one derive the values of the conduction- and valence-band discontinuity between wurtzite and zinc-blende sections from the correspondence between the TEM and PL measurements?

In order to address the first question, we start by analyzing what seems to be a twin density dependence of the PL emission. Indeed, we have observed a significant redshift (40 meV) for a twin density varying from 50 to $500 \mathrm{twins} / \mu \mathrm{m}$. Various causes may be responsible for this. For example, it is known that the binding energy of excitons confined in quantum-well-like structures increases. ${ }^{35}$ However, in GaAs,
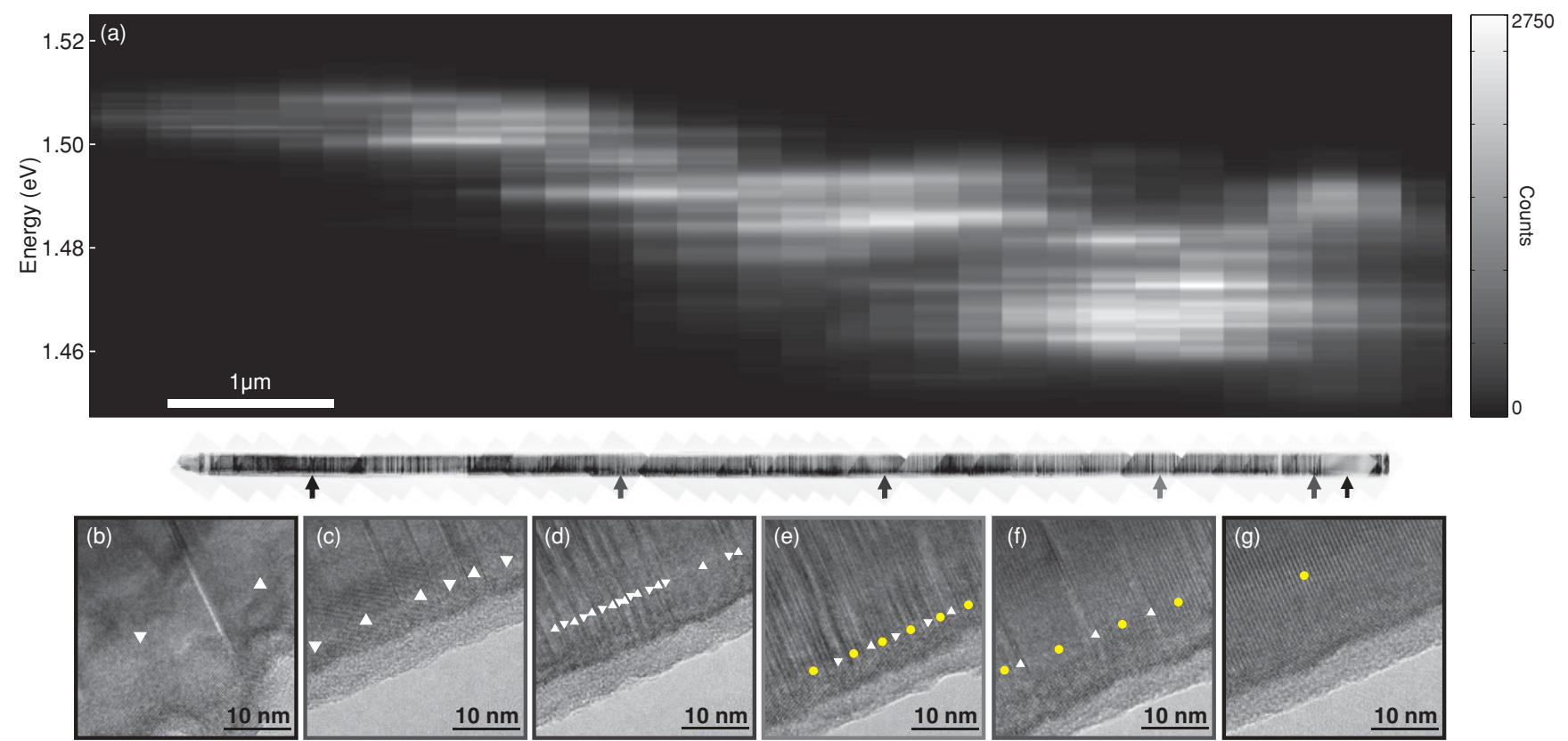

FIG. 7. (Color online) (a) Mapping of PL spectra along the length of the GaAs wurtzite/zinc-blende heterostructure nanowire shown below. The scale bar applies to both the PL mapping and the TEM. (b)-(g) HRTEM micrographs showing the crystalline phases at the positions of the nanowire indicated by the respectively colored arrows. Filled yellow circles mark the wurtzite phase while filled triangles $(\boldsymbol{\nabla}, \mathbf{\Delta})$ indicate the zinc-blende phase. 

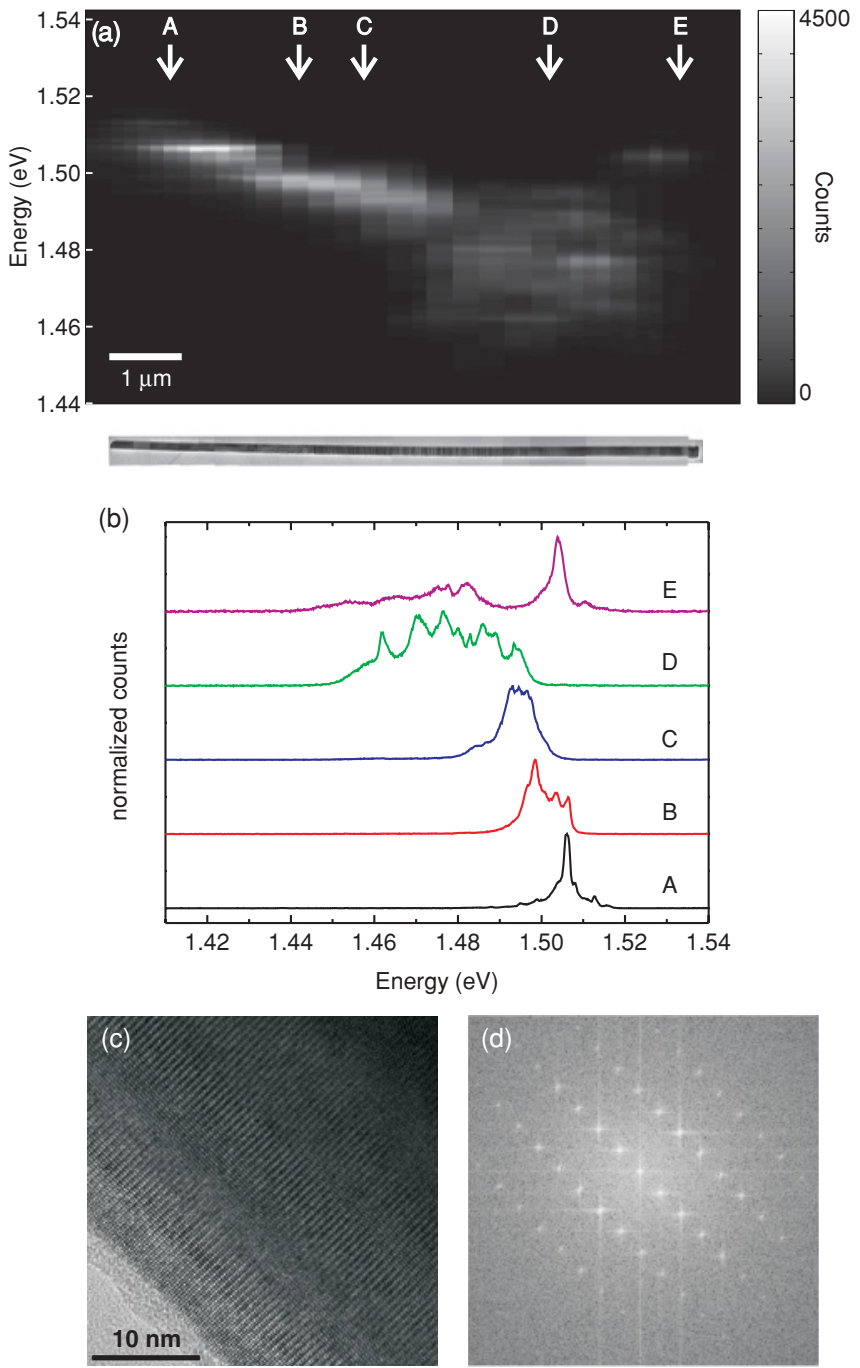

FIG. 8. (Color online) (a) PL mapping and TEM reconstruction of another wurtzite/zinc-blende heterostructure GaAs nanowire from the sample. The scale bar applies to both the PL mapping and the TEM. (b) Individual PL spectra of the five positions marked in (a). All spectra are normalized in intensity. (c) HRTEM micrograph of region $\mathrm{E}$ of the nanowire. (d) Power spectrum of (c).

even in the ideal 2D confinement limit, this could not account for a shift of more than $\sim 12 \mathrm{meV}$ at most. ${ }^{35}$ It is known that a change of effective mass within the quantum-well plane could further increase the exciton binding energies. ${ }^{36}$ However, the in-plane effective masses are not expected to change so significantly in wurtzite GaAs. ${ }^{33}$ Most importantly, the presumably type-II nature of the transition actually leads to a separation of electrons and holes, thus diminishing the Coulomb interaction between the carriers. Moreover, a type-II superlattice of single wurtzite layers in the zinc-blende sections with varying superlattice periodicity cannot be an explanation because it would lead to a blueshift instead of a redshift. Finally, we must consider the possible correlation between the twin density and the appearance of single structures with several consecutive twins, corresponding to thin sections of the wurtzite phase. The occurrence of one twin is often illustrated as 1 monolayer (ML) of wurtzite. Multiple consecutive twinning leads to the formation of thicker wurtzite segments, which could account for the PL peaks below the GaAs band gap.

For a relatively homogeneous twin density over a length of several hundreds of nanometers, the twin density in that region gives directly the probability $p$ for a twinning event to occur during nucleation of a GaAs atomic layer in the (111) direction:

$$
p=\frac{a \rho}{\sqrt{3}},
$$

where $a$ is the cubic GaAs lattice constant and $\rho$ is the density of twins in the region of the nanowire. Assuming statistical independence of the twinning process ${ }^{37}$ and the law of large numbers, the probability of $n$ subsequent twinning events to occur is given by $p_{n}=p^{n}(1-p)$. We can therefore derive the average distance between such occasional segments consisting of $n$ consecutive twins:

$$
d_{n}=\frac{a}{\sqrt{3} p_{n}} .
$$

In order to reveal the existence of multiple twinning and formation of thin segments of wurtzite, we examined each of the HRTEM micrographs along the nanowire. We found sections formed by $2-5$ ML of wurtzite in regions with increasing twin density. HRTEM micrographs corresponding to $n=2$ and $n=4$ are shown in Figs. 9(a) and 9(b). The
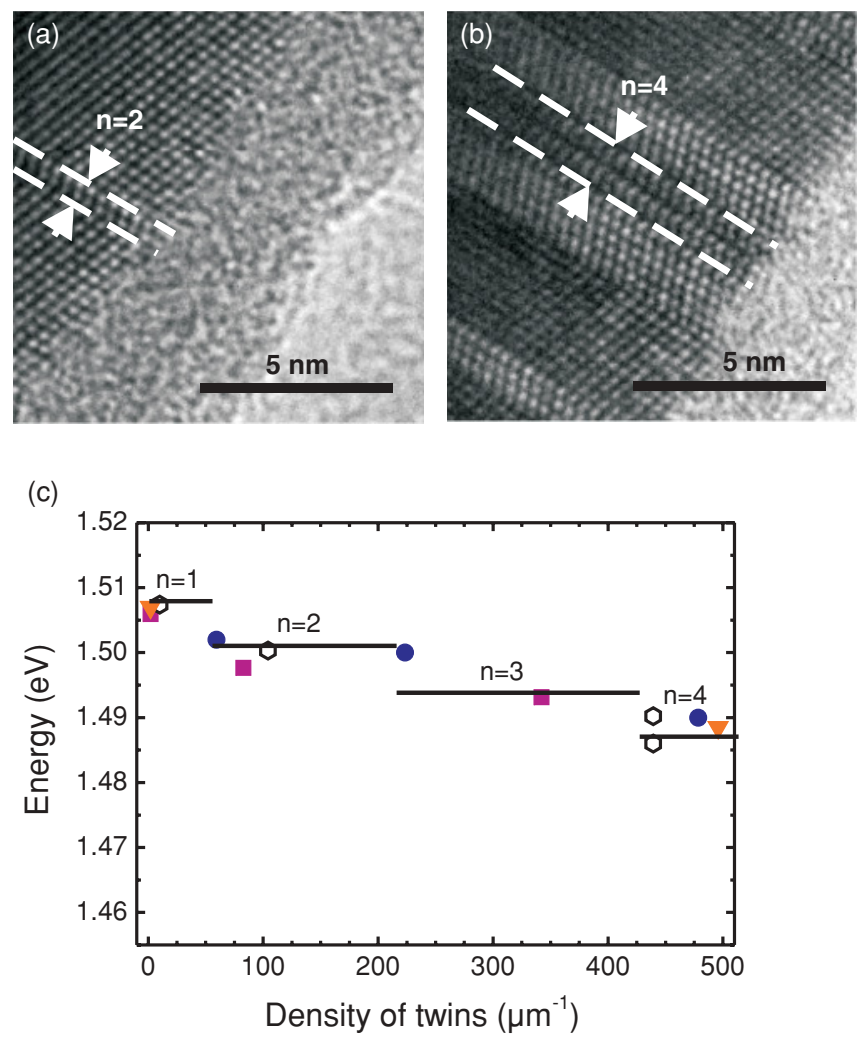

FIG. 9. (Color online) HRTEM micrographs showing the occurrence of (a) $n=2$ consecutive twins in a region with $<85$ twins $/ \mu \mathrm{m}$ and (b) $n=4$ in a region with $>400$ twins $/ \mu \mathrm{m}$. (c) Correlation of the lowest PL transition energy to the density of rotational twins in the corresponding part of the nanowire. The symbols show data obtained from four different nanowires. The black line shows the transition energies for wurtzite segments formed by $n=1-4$ consecutive twins. 
(a)

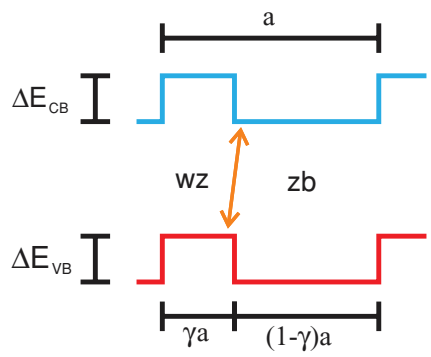

(b)
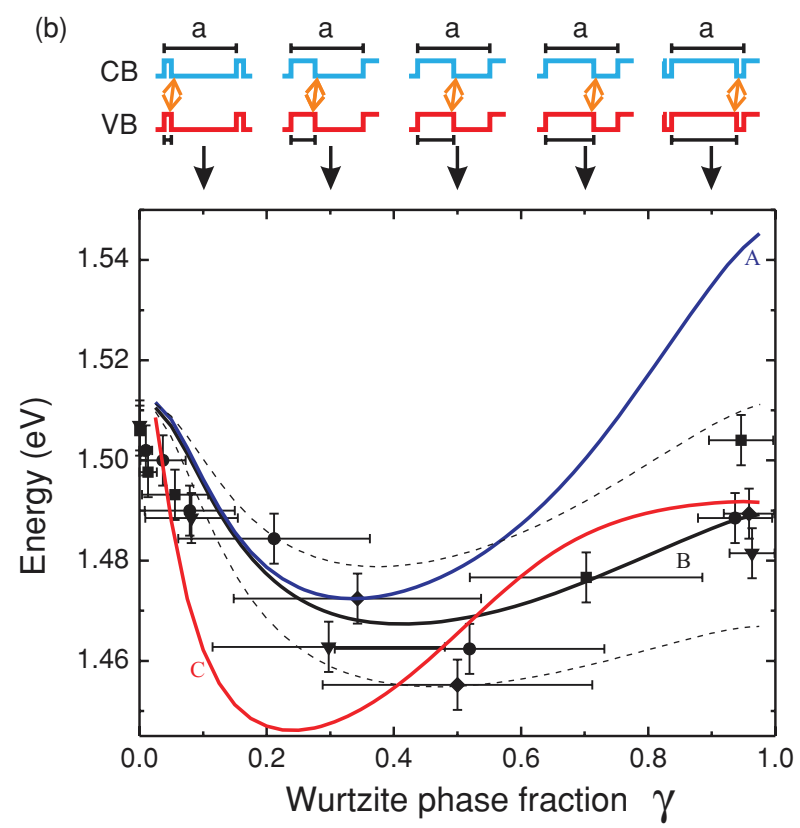

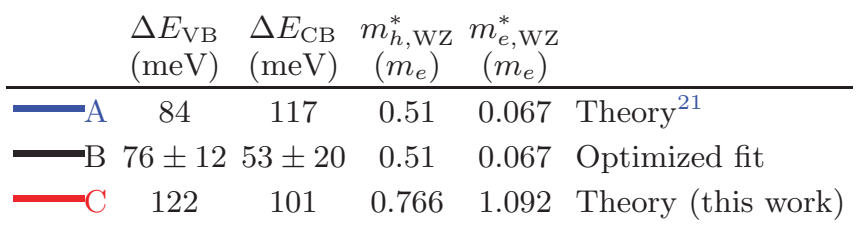

FIG. 10. (Color online) (a) Schematic of the type-II homogeneous SL model with wurtzite phase fraction $\gamma$ and periodicity $a=13 \mathrm{~nm}$. (b) Lowest PL transition energy as a function of the fraction of wurtzite phase $\gamma$ in the corresponding part of the nanowire (symbols). The colored lines show results from model calculations. The dashed black lines give the prediction bounds of the model fit to the experimental data.

position of these sections corresponds to regions in the nanowire with high PL intensity. In Fig. 9(c) we plot the PL peak position as a function of the twin density of the nanowire section. Low-temperature PL is very sensitive to the presence of such inhomogeneities with lower recombination energies. The majority of photogenerated carriers diffuse to the wurtzite quantum wells (lower energy) where they recombine. The linewidths of the peaks are extremely sharp with a full width at half maximum (FWHM) below $2.5 \mathrm{meV}$, which further corroborates our explanation [Fig. 8(b)]. We also plot the position of the peaks observed in positions where twin stacks with $n$ between 1 and 4 are observed by HRTEM. Clearly, the redshift in the PL peaks is correlated with the existence of consecutive twins and the existence of 1-, 2-, and 3-ML-thick single wurtzite regions.

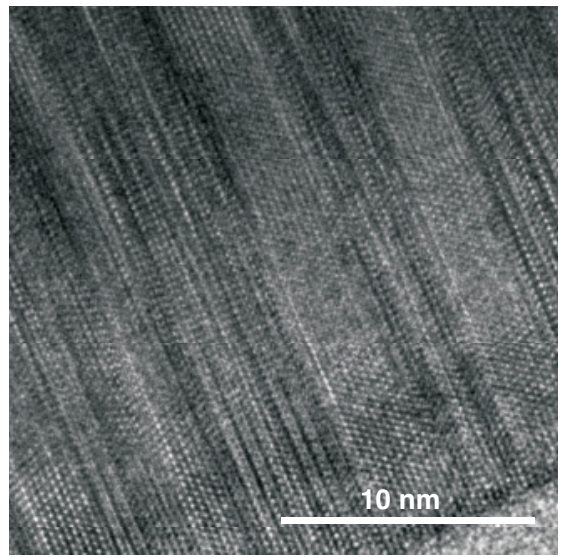

FIG. 11. HRTEM micrograph of the largest segment of the nanowire with regions composed of equal fractions of the zinc-blende and wurtzite phases, each $\sim 6.5 \mathrm{~nm}$ in length along the nanowire axis.

In the last third of the nanowire, segments of wurtzite appear regularly, as shown in Fig. 7(e). In the PL mapping, multiple peaks are observed from 1.49 down to $1.455 \mathrm{eV}$. The final part on the right-hand end of the nanowire is composed of pure wurtzite GaAs material segments with a length of up to $290 \mathrm{~nm}$ [see Figs. 7(f) and 7(g)], with small insertions of the zinc-blende structure. This region shows again PL emission above $1.49 \mathrm{eV}$. In order to address the question of whether the band offsets between wurtzite and zinc-blende GaAs phases can be determined, we plot the PL peaks as a function of the wurtzite phase fraction $\gamma$ in Fig. 10. The horizontal error bars represent the microscopic variations of the actual nonperiodic heterostructure from an ideal homogeneous superlattice. Peaks at $1.455 \mathrm{eV}$ correspond to regions of the nanowire exhibiting $\sim 50: 50$ composition of the wurtzite and zinc-blende phases. Peaks at energies higher than $1.455 \mathrm{eV}$ correspond to sections in the nanowire richer in one of the two phases. We point out that the highest-energy PL peaks in the region with pure wurtzite correspond to a band gap of $1.504 \mathrm{eV}$; we never
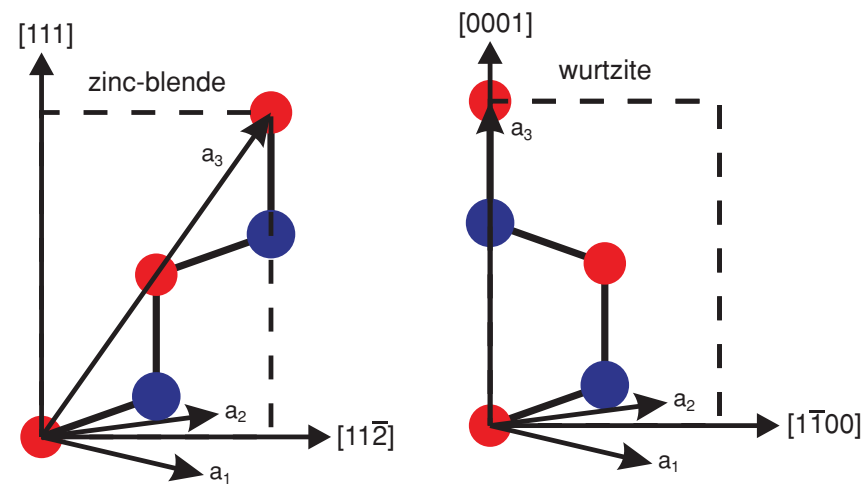

FIG. 12. (Color online) The structures used in the calculations for the zinc-blende and wurtzite lattices, with lattice vectors $\mathbf{a}_{1}, \mathbf{a}_{2}, \mathbf{a}_{3}$. The larger (blue) and smaller (red) circles denote the two types of ions and the [111] and [11 $\overline{2}]$ crystallographic directions in the zinc-blende lattice, as well as the [0001] and [1100] crystallographic direction in the wurtzite lattice are indicated. 
observed PL at energies above the free exciton of zinc-blende GaAs.

Finally, we propose a model to explain the PL emission in the last third of the nanowire. To this end, we calculate the miniband formation of a homogeneous superlattice (SL) with type-II band alignment and band offsets $\Delta E_{\mathrm{CB}}$ and $\Delta E_{\mathrm{VB}}$, shown in Fig. 10(a), by using a Kronig-Penney model. The overall lattice periodicity was set to $a_{\mathrm{SL}}=13 \mathrm{~nm}$, which is the widest periodicity observed by TEM in the region of $50: 50$ phase composition (see Fig. 11). The largest periodicities in the structure correspond to the lowest recombination energies. Low-temperature $\mu \mathrm{PL}$ is most sensitive to the transitions with the lowest recombination energies. The calculations were performed for various valence- and conduction-band offsets and are plotted in Fig. 10(b), varying the relative thickness of the wurtzite and zinc-blende segments in the superlattice and considering the experimental band gap of zinc-blende GaAs in all cases. We want to note that the calculations are neglecting modifications of the band structure from strain ${ }^{38}$ and spontaneous polarization of the hexagonal phase. ${ }^{39}$ As a first attempt to provide a quantitative explanation, we use the values $\Delta E_{\mathrm{CB}}=117 \mathrm{meV}$ and $\Delta E_{\mathrm{VB}}=84 \mathrm{meV}$ obtained in earlier theoretical work, ${ }^{21}$ and effective masses in wurtzite GaAs identical to the experimental ones for zinc-blende GaAs. The result is shown in Fig. 10(b). Clearly, there is a disagreement between the theory and the experimental data for sections of the nanowire with a large wurtzite content. By least-square fitting of the experimental data with the periodic superlattice model, we can estimate $\Delta E_{\mathrm{CB}}=53 \pm 20 \mathrm{meV}$ and $\Delta E_{\mathrm{VB}}=76 \pm 12 \mathrm{meV}$.

\section{Calculation of the band offset and effective masses}

In order to obtain more accurate band offsets and effective mass values, we performed electronic structure calculations in the context of the density functional theory (DFT) $)^{42}$ using VASP. ${ }^{43}$ For the full band structure throughout the Brillouin zone (BZ), we employed the standard local density approximation (LDA), ${ }^{44,45}$ with corresponding pseudopotentials. It is well known that this type of approach does not reproduce well the unoccupied part of the electronic spectrum and the energy band gap. Recently, it was shown that both the band gap $^{46}$ and the excitation energies ${ }^{47}$ are vastly improved by applying a screened Hartree-Fock hybrid-exchange functional (HSE06). ${ }^{48,49}$ However, such calculations owing to their high computational cost can be performed only for selected highsymmetry points in the BZ. Our approach then consists of performing a full band-structure calculation with the LDA method and using the HSE06 results to correct the excited states, by applying a uniform shift equal to the band-gap correction for the zinc-blende structure; we are fully aware that such a "scissor operator" approach may not be adequate, ${ }^{50}$ but for the bands of interest this approach gives very good results, as explained below. For the hybrid-exchange calculations, we employed the PBE exchange-correlation functional in the DFT part $^{51}$ and projector augmented wave $(\mathrm{PAW})^{52}$ potentials to represent the ions. We use a unit cell that has the same number of atoms in the zinc-blende and wurtzite lattices, by doubling one of the lattice vectors of the former, as shown in Fig. 12. We used plane-wave energy cutoffs of $326 \mathrm{eV}$ for the LDA and $400 \mathrm{eV}$ for the HSE06 calculations, and a reciprocal space Monkhorst-Pack grid of $8 \times 8 \times 4 k$ points. ${ }^{53}$ We used the experimental in-plane lattice constant of zinc-blende (3.99 $\AA$ ) corresponding to bulk $a=5.65 \AA$. This is very close to the calculated ones ( $3.95 \AA$ ) for both the zinc-blende and wurtzite phases. For this we neglected the effect of strain and influence of band structure.

The results of the calculations are collected in Table I and the band structures for the zinc-blende and wurtzite phases are shown in Fig. 13, along the important directions, that is, [111] for zinc-blende phases and [0001] for wurtzite phases. We can calculate the band structure of this interface and assign each band to the relevant wurtzite or zinc-blende phase, based on density distribution and similarity to bands of the bulk zinc-blende and wurtzite structure. In this way we can get information of band alignment and/or offsets of this interface. It is clear from the plot in Fig. 13 that along these directions in reciprocal space, the uniform shift of excited LDA states (open black circles) by an amount equal to the band-gap correction for the zinc-blende phase, brings them to excellent agreement with the calculated HSE06 results (filled red circles). The calculated band offsets are $\Delta E_{\mathrm{CB}}=101 \mathrm{meV}$ and $\Delta E_{\mathrm{VB}}=$ $122 \mathrm{meV}$, and effective masses along the [0001] wurtzite orientation are $m_{h, \mathrm{WZ}}^{*}=0.766 m_{e}$ and $m_{e, \mathrm{WZ}}^{*}=1.092 m_{e}$; the

TABLE I. The calculated energy band gaps and band offsets: LDA and HSE06 refer to the present work (see the text for details). For comparison, we also include the results of earlier work by using ab initio calculations (Refs. 21, 40, and 41) or an empirical pseudopotential method (Ref. 33). Experimental results for band offsets are from the optimal fit shown in Fig. 10. $\varepsilon_{\text {gap-ZB }}$ is the band gap of the zinc-blende phase while $\Delta \varepsilon_{\text {gap }}=\varepsilon_{\text {gap-WZ }}-\varepsilon_{\text {gap-ZB }}$ corresponds to the difference in band gap between the zinc-blende and wurtzite phases.

\begin{tabular}{lcccc}
\hline \hline & $\varepsilon_{\text {gap }-\mathrm{ZB}}(\mathrm{eV})$ & $\Delta \varepsilon_{\text {gap }}(\mathrm{eV})$ & $\Delta E_{\mathrm{VB}}(\mathrm{meV})$ & $\Delta E_{\mathrm{CB}}(\mathrm{meV})$ \\
\hline DFT-LDA (Ref. 21) & 0.614 & +0.033 & 84 & 117 \\
DFT-LDA (Ref. 40) & 0.44 & +0.03 & - & - \\
DFT-GW (Ref. 41) & 1.133 & +0.218 & - & - \\
EP (Ref. 33) & $1.519^{\mathrm{a}}$ & -0.016 & 79.2 & 63.2 \\
DFT-LDA & 0.351 & -0.044 & 114 & 70 \\
DFT-HSE06 & 1.405 & -0.021 & 122 & 101 \\
experiment & 1.51 & -0.023 & $76 \pm 12$ & $53 \pm 20$ \\
\hline \hline
\end{tabular}

${ }^{\mathrm{a}}$ Based on a fit to the experimental zinc-blende band gap. 


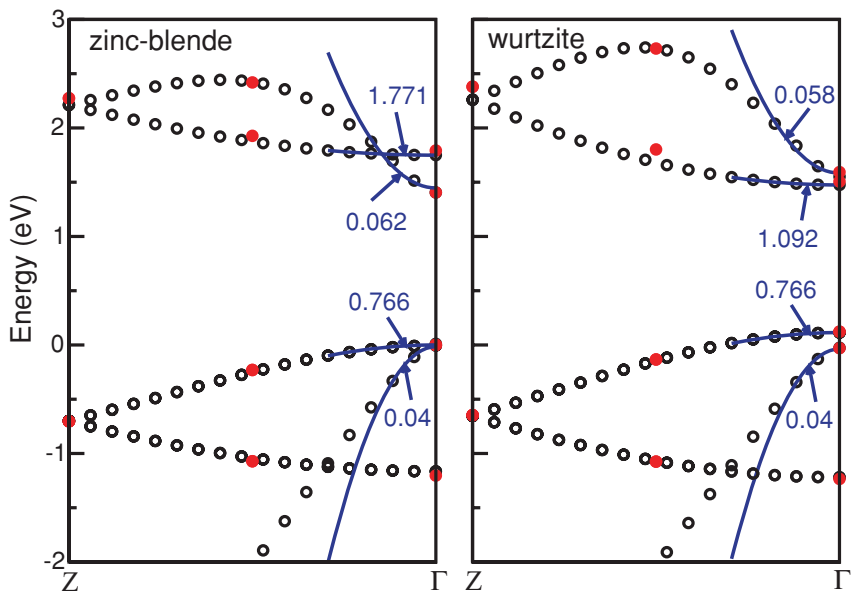

FIG. 13. (Color online) The band structures of the zinc-blende and wurtzite lattices along the $\mathrm{Z} \Gamma$ direction in the Brillouin zone. Open black circles are the results of the LDA calculations, red filled circles are the results of HSE06 calculations, and blue lines are quadratic fits near $\Gamma$. The values in blue indicate the effective masses in units of $m_{e}$ obtained from the quadratic fits.

corresponding values for the [111] zinc-blende orientation are $m_{h, \mathrm{ZB}}^{*}=0.766 m_{e}$ and $m_{e, \mathrm{ZB}}^{*}=0.062 m_{e}$. The essential difference is in the electron effective mass, which comes from the folding of the bands along the [0001] wurtzite orientation, as seen in Fig. 13. The corresponding superlattice model curve using the calculated band offsets and wurtzite effective masses is shown in Fig. 10(b). Our first-principles calculations indicate that the band gap of wurtzite GaAs is $21 \mathrm{meV}$ lower as compared to the band gap of the zinc-blende phase and are in good agreement with the experimental data. Our experimentally determined band offsets are also in excellent agreement with band offsets recently calculated by De and Pryor ${ }^{33}$ using an empirical pseudopotential method (see Table I).

\section{CONCLUSIONS}

In conclusion, we have presented results on a technique that enables the experimental direct correlation between confocal $\mu \mathrm{PL}$ measurements and the structural characterization with HRTEM of nanowires consisting of nearly $100 \%$ wurtzite and presenting wurtzite/zinc-blende polytypism. The observations are in good agreement with theoretical predictions for the band gap and band offsets between the wurtzite and zincblende phases of GaAs. In wurtzite GaAs nanowires, we observe PL always below $1.52 \mathrm{eV}$. Taking into account the electronic confinement, the results indicate that the electronic band gap of the wurtzite GaAs phase is slightly smaller than zinc-blende GaAs, with an estimated $\varepsilon_{\text {gap }-\mathrm{wZ}}=$ $1.50 \mathrm{eV}$.

The results on the polytypic nanowires are multiple. First, we correlate the appearance of wurtzite sections with the probability of twin formation. Then, we link the position of the luminescence with the stacking sequence of the wurtzite and zinc-blende phases. Owing to the fact that the actual nanowire heterostructure is nonperiodic, an exact calculation for the optical transitions would require a model including the complete stacking sequence of each nanowire with atomic precision; it is extremely challenging to determine this sequence experimentally. By using a more simple periodic superlattice model, we have demonstrated that the experimental findings can be well explained despite the drastic simplifications of the model; we have also determined an experimental estimation for the band offsets of $\Delta E_{\mathrm{CB}}=53 \pm 20 \mathrm{meV}$ and $\Delta E_{\mathrm{VB}}=76 \pm 12 \mathrm{meV}$. These band offsets correspond to a band gap for wurtzite GaAs of $\sim 23 \mathrm{meV}$ lower compared to the band gap of the zinc-blende phase. This is in excellent agreement with our results on pure wurtzite GaAs nanowires, other experiments, ${ }^{27}$ our first-principles calculations, and other recent band-structure calculations. ${ }^{33}$ Furthermore, a slightly smaller band gap of the wurtzite phase of GaAs is supported by our general observation that even though both nanowire samples contain large amounts of wurtzite GaAs, we have never observed PL emission above the band gap of zinc-blende GaAs.

\section{ACKNOWLEDGMENTS}

The authors thank G. Abstreiter, T. Garma, D. Spirkoska, and M. Bichler for their experimental support and discussions. We thank A. Petroutchik and L. T. Baczewski for the preparation of the Au-covered substrates for nanowire growth. This research was supported by Marie Curie Excellence Grant SENFED, the DFG excellence cluster Nanosystems Initiative Munich, as well as SFB 631 and ERC Starting Grant Upcon. This work was partially supported by the Spanish Government projects Consolider Ingenio 2010 CSD2009 00013 IMAGINE and CSD2009 00050 MULTICAT. J.A. acknowledge funding from the Spanish CSIC project NEAMAN and MICINN project MAT2010-15138 (COPEON). The authors would like to thank the TEM facilities in the Interdisciplinmulary Center for Electron Microscopy in Lausanne and in Barcelona. E.R. acknowledges financial support from the ERA Nanoscience Project QOptInt. E.R. and D.S. acknowledge support via SFB 689.

\footnotetext{
*anna.fontcuberta-morral@epfl.ch

${ }^{1}$ Y. Cui and C. M. Lieber, Science 291, 851 (2001).

${ }^{2}$ L. Samuelson, M. T. Björk, K. Deppert, M. Larsson, B. J. Ohlsson, N. Panev, A. I. Persson, N. Sköld, C. Thelander, and L. R. Wallenberg, Physica E 21, 560 (2004).

${ }^{3}$ Y. Cui, Z. Zhong, D. Wang, W. U. Wang, and C. M. Lieber, Nano Lett. 3, 149 (2003).
}

${ }^{4}$ M. T. Bjork, B. J. Ohlsson, T. Sass, A. I. Persson, C. Thelander, M. H. Magnusson, K. Deppert, L. R. Wallenberg, and L. Samuelson, Appl. Phys. Lett. 80, 1058 (2002).

${ }^{5}$ W. U. Wang, C. Chen, K.-h. Lin, Y. Fang, and C. M. Lieber, Proc. Natl. Acad. Sci. USA 102, 3208 (2005).

${ }^{6}$ X. Duan, Y. Huang, Y. Cui, J. Wang, and C. M. Lieber, Nature (London) 409, 66 (2001). 
${ }^{7}$ C. K. Chan, H. Peng, G. Liu, K. Mcllwrath, X. F. Zhang, R. A. Huggins, and Y. Cui, Nat. Nanotechnol. 3, 31 (2008).

${ }^{8}$ B. Tian, X. Zheng, T. J. Kempa, Y. Fang, N. Yu, G. Yu, J. Huang, and C. M. Lieber, Nature (London) 449, 885 (2007).

${ }^{9}$ B. M. Kayes, H. A. Atwater, and N. S. Lewis, J. Appl. Phys. 97, 114302 (2005).

${ }^{10}$ L. J. Lauhon, M. S. Gudiksen, D. Wang, and C. M. Lieber, Nature (London) 420, 57 (2002).

${ }^{11}$ M. S. Gudiksen, L. J. Lauhon, J. Wang, D. C. Smith, and C. M. Lieber, Nature (London) 415, 617 (2002).

${ }^{12}$ A. Fontcuberta i Morral, D. Spirkoska, J. Arbiol, M. Heigoldt, J. R. Morante, and G. Abstreiter, Small 4, 899 (2008).

${ }^{13}$ M. Heigoldt, J. Arbiol, D. Spirkoska, J. M. Rebled, S. Conesa-Boj, G. Abstreiter, F. Peiro, J. R. Morante, and A. Fontcuberta i Morral, J. Mater. Chem. 19, 840 (2009).

${ }^{14}$ M. Heiss, A. Gustafsson, S. Conesa-Boj, F. Peiro, J. R. Morante, G. Abstreiter, J. Arbiol, L. Samuelson, and A. Fontcuberta i Morral, Nanotechnology 20, 075603 (2009).

${ }^{15}$ F. M. Ross, Nat. Nanotechnol. 4, 17 (2009).

${ }^{16}$ P. Caroff, K. A. Dick, J. Johansson, M. E. Messing, K. Deppert, and L. Samuelson, Nat. Nanotechnol. 4, 50 (2009).

${ }^{17}$ R. E. Algra, M. A. Verheijen, M. T. Borgstrom, L.-F. Feiner, G. Immink, W. J. P. van Enckevort, E. Vlieg, and E. P. A. M. Bakkers, Nature (London) 456, 369 (2008).

${ }^{18}$ C. H. Park, B.-H. Cheong, K.-H. Lee, and K. J. Chang, Phys. Rev. B 49, 4485 (1994).

${ }^{19}$ S. Strite and H. Morkoç, J. Vac. Sci. Technol. B 10, 1237 (1992).

${ }^{20}$ F. M. Davidson, D. C. Lee, D. D. Fanfair, and B. A. Korgel, J. Phys. Chem. C 111, 2929 (2007).

${ }^{21}$ M. Murayama and T. Nakayama, Phys. Rev. B 49, 4710 (1994).

${ }^{22}$ J. Bao, D. C. Bell, F. Capasso, J. B. Wagner, T. Mårtensson, J. Trägårdh, and L. Samuelson, Nano Lett. 8, 836 (2008).

${ }^{23}$ K. Pemasiri, M. Montazeri, R. Gass, L. M. Smith, H. E. Jackson, J. Yarrison-Rice, S. Paiman, Q. Gao, H. H. Tan, C. Jagadish, X. Zhang, and J. Zou, Nano Lett. 9, 648 (2009).

${ }^{24}$ N. Akopian, G. Patriarche, L. Liu, J.-C. Harmand, and V. Zwiller, Nano Lett. 10, 1198 (2010).

${ }^{25}$ T. B. Hoang, A. F. Moses, H. L. Zhou, D. L. Dheeraj, B. O. Fimland, and H. Weman, Appl. Phys. Lett. 94, 133105 (2009).

${ }^{26}$ F. Martelli, M. Piccin, G. Bais, F. Jabeen, S. Ambrosini, S. Rubini, and A. Franciosi, Nanotechnology 18, 125603 (2007).

${ }^{27}$ M. Moewe, L. C. Chuang, S. Crankshaw, C. Chase, and C. ChangHasnain, Appl. Phys. Lett. 93, 023116 (2008).

${ }^{28}$ D. Spirkoska, J. Arbiol, A. Gustafsson, S. Conesa-Boj, F. Glas, I. Zardo, M. Heigoldt, M. H. Gass, A. L. Bleloch, S. Estrade, M. Kaniber, J. Rossler, F. Peiro, J. R. Morante, G. Abstreiter, L. Samuelson, and A. Fontcuberta i Morral, Phys. Rev. B 80, 245325 (2009).
${ }^{29}$ J. Arbiol, S. Estrade, J. D. Prades, A. Cirera, F. Furtmayr, C. Stark, A. Laufer, M. Stutzmann, M. Eickhoff, M. H. Gass, A. L. Bleloch, F. Peiro, and J. R. Morante, Nanotechnology 20, 145704 (2009).

${ }^{30}$ S. K. Lim, M. Brewster, F. Qian, Y. Li, C. M. Lieber, and S. Gradečak, Nano Lett. 9, 3940 (2009).

${ }^{31}$ R. S. Wagner and W. C. Ellis, Nano Lett. 4, 89 (1964).

${ }^{32}$ A. Rudolph, M. Soda, M. Kiessling, T. Wojtowicz, D. Schuh, W. Wegscheider, J. Zweck, C. Back, and E. Reiger, Nano Lett. 9, 3860 (2009).

${ }^{33}$ A. De and C. E. Pryor, Phys. Rev. B 81, 155210 (2010).

${ }^{34}$ S. Birner, T. Zibold, T. Andlauer, T. Kubis, M. Sabathil, A. Trellakis, and P. Vogl, IEEE Trans. Electron Devices 54, 2137 (2007).

${ }^{35}$ R. C. Miller, D. A. Kleinman, W. T. Tsang, and A. C. Gossard, Phys. Rev. B 24, 1134 (1981).

${ }^{36}$ C. Ropers, M. Wenderoth, L. Winking, T. C. G. Reusch, M. Erdmann, R. G. Ulbrich, M. Grochol, F. Grosse, R. Zimmermann, S. Malzer, and G. H. Döhler, Phys. Rev. B 75, 115317 (2007).

${ }^{37}$ J. Johansson, L. S. Karlsson, C. P. T. Svensson, T. Martensson, B. A. Wacaser, K. Deppert, L. Samuelson, and W. Seifert, Nat. Mater. 5, 574 (2006).

${ }^{38}$ I. Zardo, S. Conesa-Boj, F. Peiro, J. R. Morante, J. Arbiol, E. Uccelli, G. Abstreiter, and A. Fontcuberta i Morral, Phys. Rev. B 80, 245324 (2009).

${ }^{39}$ F. Bernardini, V. Fiorentini, and D. Vanderbilt, Phys. Rev. B 56, R10024 (1997).

${ }^{40}$ C.-Y. Yeh, S.-H. Wei, and A. Zunger, Phys. Rev. B 50, 2715 (1994).

${ }^{41}$ Z. Zanolli, F. Fuchs, J. Furthmüller, U. von Barth, and F. Bechstedt, Phys. Rev. B 75, 245121 (2007).

${ }^{42}$ W. Kohn and L. J. Sham, Phys. Rev. 140, A1133 (1965).

${ }^{43}$ G. Kresse and J. Furthmüller, Phys. Rev. B 54, 11169 (1996).

${ }^{44}$ D. M. Ceperley and B. J. Alder, Phys. Rev. Lett. 45, 566 (1980).

${ }^{45}$ J. P. Perdew and A. Zunger, Phys. Rev. B 23, 5048 (1981).

${ }^{46}$ M. Marsman, J. Paier, A. Stroppa, and G. Kresse, J. Phys. Condens. Matter 20, 064201 (2008).

${ }^{47}$ J. Paier, M. Marsman, and G. Kresse, Phys. Rev. B 78, 121201 (2008).

${ }^{48}$ J. Heyd, G. E. Scuseria, and M. Ernzerhof, J. Chem. Phys. 118, 8207 (2003).

${ }^{49}$ A. V. Krukau, O. A. Vydrov, A. F. Izmaylov, and G. E. Scuseria, J. Chem. Phys. 125, 224106 (2006).

${ }^{50}$ I. N. Remediakis and E. Kaxiras, Phys. Rev. B 59, 5536 (1999).

${ }^{51}$ J. P. Perdew, K. Burke, and M. Ernzerhof, Phys. Rev. Lett. 77, 3865 (1996).

${ }^{52}$ P. Blöchl, C. Först, and J. Schimpl, Bull. Mater. Sci. 26, 33 (2003).

${ }^{53}$ H. J. Monkhorst and J. D. Pack, Phys. Rev. B 13, 5188 (1976). 